\title{
The Influence of Clinical and Patient-Reported Outcomes on Post-surgery Satisfaction in Cholecystectomy Patients
}

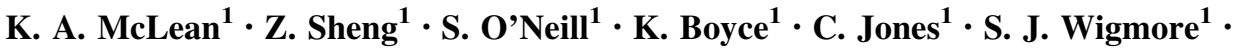 \\ E. M. Harrison ${ }^{1}$
}

Published online: 9 March 2017

(c) The Author(s) 2017. This article is published with open access at Springerlink.com

\begin{abstract}
Background Patient-reported outcomes (PROMs) and post-operative satisfaction have become a growing focus of surgical outcome evaluation and are considered key components of the movement towards patient-centred care. The aim was to compare the association of traditional clinical outcome measures and PROMs with post-surgery satisfaction in cholecystectomy patients.

Methods Patients who had undergone elective or emergency cholecystectomy for gallstone disease were sent validated PROM questionnaires, and telephone follow-up was performed in all cases. Categorical data were compared with the Chi-square and Fisher's exact tests. Satisfaction was investigated using a "top-box" approach, and multivariable logistic regression was performed for factors significantly $(p<0.05)$ associated with satisfaction in univariable analyses.

Results A total of 234 patients underwent cholecystectomy between 1 March 2014 and 1 May 2014, and 147 patients (63\%) completed the questionnaire. 104/147 (71\%) reported being "very satisfied" with their surgical outcome. In univariable analyses, satisfaction showed significant association with an absence of hospital-recorded 30-day complications (OR $=4.11,95 \%$ CI 1.29-13.84), but not re-attendance, readmission, or length of stay. In a multiple regression analysis, no traditional clinical outcome measures were associated with satisfaction. By contrast, selfperceived health $(\mathrm{OR}=4.04,95 \% \mathrm{CI} 1.44-11.86)$, the absence of patient-reported wound pain $(\mathrm{OR}=6.11,95 \% \mathrm{CI}$ 1.83-21.74), and a return to normal leisure activities $(\mathrm{OR}=11.14,95 \%$ CI 2.61-55.26) were associated with satisfaction.

Conclusion PROMs are the major determinants of patient satisfaction following cholecystectomy. When assessing outcomes following cholecystectomy, the measurement of clinical outcomes alone is inadequate and should be supplemented by the use of PROMs.
\end{abstract}

E. M. Harrison

ewen.harrison@ed.ac.uk

1 Clinical Surgery, University of Edinburgh, Royal Infirmary of Edinburgh, 51 Little France Crescent, Edinburgh EH16 4SA, Scotland

\section{Introduction}

Biliary pain secondary to cholelithiasis is among the most common gastrointestinal causes of hospitalisation [1, 2], and laparoscopic cholecystectomy is considered the "gold standard" treatment for symptomatic cholelithiasis [3].

Patient-reported outcomes measures (PROMs), including satisfaction and health-related quality of life (HRQoL), have been increasingly recognised as potentially invaluable tools in both the assessment and quality improvement in 
healthcare interventions [4]. The isolated use of traditional, objective clinical outcomes following surgery can fail to recognise factors which are important to patients, and the contribution the perspective of patients can have in healthcare appraisal. It has been said that the ultimate measure by which to judge the quality of a medical effort is whether it helps patients as they see it [5]. As such, PROMs have become a growing focus of surgical outcome evaluation [4, 6] and are considered key to the movement towards more patient-centred healthcare [7]. These also have the potential to expose the hidden burden of surgical interventions which may otherwise be self-managed or addressed in primary care.

This study aimed to determine the relationship of clinical outcome measures and PROMs with post-surgery satisfaction in a cohort of cholecystectomy patients within a 3-month post-operative period.

\section{Materials and methods}

\section{Patients/recruitment}

Consecutive patients were recruited prospectively and underwent cholecystectomy (open or laparoscopic) at the Royal Infirmary of Edinburgh from the 1 March 2014 to the 1 May 2014. This included all patients over 18 years and excluded cholecystectomies due to known gallbladder cancer or resulting from another surgical procedure.

\section{Data collection}

The electronic patient record was used to obtain sociodemographic, clinical, and contact information. PROMs were assessed using combined general (EQ-5D-5L [8]) and condition-specific $[9,10]$ questionnaires (Appendix 1) and focussed on post-operative symptoms, post-operative function, and satisfaction. A questionnaire was sent via post to the home address of each patient and was supplemented by a telephone interview. The follow-up interviews were performed by independent individuals trained for the purposes of the study (KAM, ZS) and had no knowledge of the details of surgery or recovery. These were completed between 1- and 3-month post-cholecystectomy, and three separate attempts were made to contact each patient by telephone. Institutional ethical approval was obtained and informed consent gained from each patient contacted (Appendix 2).

\section{Statistical analyses}

The questionnaire responses were assessed using either a binary or five-point Likert scale and combined into dichotomous categories for analytic purposes. The patient satisfaction variable was investigated using a "top-box" approach comparing those who were "very satisfied" (5) to those who were not "very satisfied" (1-4), as previously described [11-13]. All other Likert variables were aggregated into "low" (1-2), and "high" (3-5) responses. In addition, we summarised the association between paired hospital-recorded and patient-reported 30-day complication rates using a McNemar odds ratio.

Continuous data were summarised as a mean and range and compared using $t$ tests. Categorical data were crosstabulated and differences in proportions tested using the Chi-square or Fisher's exact test. Where appropriate, an odds ratio (OR) and $95 \%$ confidence interval $(95 \% \mathrm{CI})$ were calculated. Multivariable logistic regression was performed with variables identified as significantly associated with satisfaction on univariable analysis. R Studio v2.1 (R Foundation for Statistical Computing) was used for statistical analyses, and $p<0.05$ was considered to be statistically significant.

\section{Results}

A total of 234 consecutive patients were recruited during the study period. Of these, $165(71 \%)$ responded to the questionnaire by either post or telephone; however, 18 $(8 \%)$ questionnaires were returned incomplete and so were excluded. Six (3\%) refused participation, 63 (27\%) did not respond, and so 147 (63\%) were included in the analyses. The characteristics of respondent and non-respondents were similar (Table 1) with respondents being older than non-respondents (57.7 years vs. 48.5 years, $p<0.001)$ and with a higher ASA classification $(p=0.044)$. One hundred and four respondents (71\%) reported being "very satisfied" with the outcome of their cholecystectomy, 37 (25\%) being "satisfied" (an overall satisfaction rate of 96\%), and $6(4 \%)$ reporting feeling "worse", "no effect", or "do not know". No baseline differences were seen between satisfaction groups (Table 2), including the operative approach utilised, type of admission (acute, delayed, or elective), or time post-operative.

When the associations between post-operative satisfaction and post-operative symptoms were explored (Table 3), the absence of patient-reported 30-day complications such as wound pain [OR: $5.98(2.39-15.60), p<0.001]$, or other symptoms [OR: $2.42(1.04-5.63), p=0.026$ ] showed significant association with high satisfaction. Furthermore, the absence of recurrent symptoms [OR: 5.08 (1.76-15.61), $p=0.001]$, or de novo heartburn [OR: 3.29 (1.24-8.86), $p=0.014]$ was also significantly associated with satisfaction.

The association of patient-reported physical, emotional, and social functionality with patient satisfaction was 
Table 1 Demographic and pre-operative characteristics of respondents and non-respondents

\begin{tabular}{|c|c|c|c|}
\hline & \multicolumn{3}{|c|}{ Patient-reported satisfaction } \\
\hline & $\begin{array}{l}\text { Respondents } \\
n=147\end{array}$ & $\begin{array}{l}\text { Non-respondents } \\
n=87\end{array}$ & $p$ value \\
\hline \multicolumn{4}{|l|}{ Demographics } \\
\hline \multicolumn{4}{|l|}{ Age (years) } \\
\hline Mean (range) & $57(22-86)$ & $49(20-84)$ & $<0.001$ \\
\hline \multicolumn{4}{|l|}{ BMI $\left(\mathrm{kg} / \mathrm{m}^{2}\right)$} \\
\hline Mean (range) & $30(18-48)$ & $29(17-42)$ & 0.380 \\
\hline \multicolumn{4}{|l|}{ Gender } \\
\hline Male & $44(30)$ & $17(19)$ & 0.091 \\
\hline Female & $103(70)$ & $70(81)$ & \\
\hline \multicolumn{4}{|c|}{ Pre-operative characteristics } \\
\hline \multicolumn{4}{|l|}{ Type of admission } \\
\hline Acute & $52(35)$ & $28(32)$ & 0.469 \\
\hline Elective & $29(20)$ & $13(15)$ & \\
\hline Delayed & $66(45)$ & $46(53)$ & \\
\hline \multicolumn{4}{|l|}{ ASA } \\
\hline ASA 1 & $57(39)$ & $45(52)$ & 0.044 \\
\hline ASA 2 & 69 (47) & $35(40)$ & \\
\hline $\mathrm{ASA} \geq 3$ & $20(14)$ & $7(8)$ & \\
\hline \multicolumn{4}{|l|}{ Indication } \\
\hline Biliary colic & $75(51)$ & $41(47)$ & 0.440 \\
\hline Cholecystitis & $42(29)$ & $28(32)$ & \\
\hline Gallstone pancreatitis & $11(8)$ & $11(13)$ & \\
\hline CBD stones & $16(11)$ & $7(8)$ & \\
\hline Other $^{\mathrm{a}}$ & $3(1)$ & $0(0)$ & \\
\hline \multicolumn{4}{|l|}{ Approach } \\
\hline Laparoscopic & $132(90)$ & $83(95)$ & 0.346 \\
\hline Open & $2(1)$ & $0(0)$ & \\
\hline Laparoscopic to open & $13(9)$ & $4(5)$ & \\
\hline
\end{tabular}

Data are $n(\%)$ unless otherwise stated

${ }^{a}$ Polyps $(n=2)$ and Acalculous $(n=1)$

explored. Satisfaction was strongly associated with a quick return to daily duties [ORs: $5.10(1.90-14.30), p<0.001$ ] and leisure activities [10.35 (3.47-35.46), $p<0.001$, together with minimal post-operative fatigue [OR: 2.91 (1.32-6.53), $p=0.005$ ] or mood alterations [OR: 3.59 (1.33-9.90), $p=0.006]$. Similarly, when evaluating current health, satisfaction was associated with the absence of pain [OR: $3.56(1.08-12.20), p=0.020]$ and high selfperceived health [OR: $5.16(2.24-12.58), p<0.001]$.

In an analysis of traditional clinical outcome measures, satisfaction was associated with the absence of hospitalrecorded 30-day complications [OR: 4.11 (1.29-13.84), $p=0.009]$, but not re-attendance, readmission, nor length of stay.

Multivariable logistic regression revealed that the absence of patient-reported wound pain [OR: 7.32
Table 2 Demographic and pre-operative characteristics of the satisfaction subgroups

$\frac{\text { Patient-reported satisfaction }}{\text { Not very satisfied Very satisfied } p \text { value }}$

$n=43 \quad n=104$

Demographics

Age (years)

$\begin{array}{llll}\begin{array}{l}\text { Mean (range) } \\ \text { BMI }\left(\mathrm{kg} / \mathrm{m}^{2}\right)\end{array} & 56(24-85) & 57(22-86) & 0.603 \\ \begin{array}{l}\text { Mean (range) } \\ \text { Gender }\end{array} & 30(21-46) & 30(18-48) & 0.580 \\ \text { Male } & 13(30) & 31(30) & 1.000 \\ \text { Female } & 30(70) & 73(70) & \end{array}$

Pre-operative characteristics

Type of admission

\begin{tabular}{llll} 
Acute & $15(35)$ & $51(49)$ & 0.255 \\
Elective & $19(44)$ & $33(32)$ & \\
Delayed & $9(21)$ & $20(19)$ & \\
ASA ${ }^{\text {a }}$ & & & \\
ASA 1 & $18(43)$ & $39(38)$ & 0.881 \\
ASA 2 & $18(43)$ & $51(49)$ & \\
ASA $\geq 3$ & $6(14)$ & $14(13)$ & \\
Indication & & & \\
Biliary colic & $23(54)$ & $52(50)$ & 0.964 \\
Cholecystitis & $12(28)$ & $30(29)$ & \\
Gallstone & $2(5)$ & $9(9)$ & \\
pancreatitis & & $12(12)$ & \\
CBD stones & $4(9)$ & $1(1)$ & \\
Other ${ }^{b}$ & $2(5)$ & $96(92)$ & 0.095 \\
Approach & & $2(2)$ & \\
Laparoscopic & $36(84)$ & $6(6)$ & \\
Open & $0(0)$ & & \\
Laparoscopic to & $7(16)$ & $29(28)$ & \\
open & & $51(49)$ & \\
Time post-operative & & & \\
$\leq 30$ days & $12(28)$ & $20(47)$ & \\
31-60 days & $11(26)$ & & \\
$>60$ days & & & \\
\hline
\end{tabular}

Data are $n(\%)$ unless otherwise stated

a Not very satisfied $n=42$

b Polyps $(n=2)$ and Acalculous $(n=1)$

(2.35-24.46), $p<0.001]$, a return to normal leisure activities [OR: 12.19 (2.87-59.83), $p=0.001$ ], and a high selfperceived opinion of current health [OR: 3.87 (1.39-11.23), $p=0.010]$ were independently associated with higher post-cholecystectomy satisfaction.

Finally, a McNemar odds ratio (mOR) was used to summarise the association in paired patient-reported and hospital-recorded 30-day complication rates (Table 4). 
Table 3 Univariate association of PROM and clinical outcomes with patient-reported satisfaction, and multivariate logistic regression of significant variables

Patient-reported satisfaction

\begin{tabular}{|c|c|c|c|c|c|}
\hline \multirow{2}{*}{$\begin{array}{l}\text { Not very } \\
\text { satisfied } n=43\end{array}$} & \multirow{2}{*}{$\begin{array}{l}\text { Very satisfied } \\
n=104\end{array}$} & \multicolumn{2}{|c|}{ Univariate association } & \multicolumn{2}{|c|}{ Multivariate logistic regression } \\
\hline & & $\overline{\mathrm{OR}(95 \% \mathrm{CI})}$ & $p$ value & OR $(95 \% \mathrm{CI})$ & $p$ value \\
\hline
\end{tabular}

\begin{tabular}{|c|c|c|c|c|c|c|}
\hline \multicolumn{3}{|l|}{ Post-operative symptoms } & & & & \\
\hline \multicolumn{7}{|l|}{ 30-day period } \\
\hline No complications & $27(63)$ & $78(75)$ & $1.77(0.77-4.05)$ & 0.161 & - & - \\
\hline No wound infection & $37(86)$ & $97(93)$ & $2.23(0.58-8.34)$ & 0.202 & - & - \\
\hline No fever & 37 (86) & $98(94)$ & $2.63(0.66-10.52)$ & 0.110 & - & - \\
\hline No wound pain & $24(56)$ & $92(88)$ & $5.98(2.39-15.60)$ & $<0.001$ & $7.32(2.35-24.46)$ & $<0.001$ \\
\hline No other symptoms & $26(60)$ & $82(79)$ & $2.42(1.04-5.63)$ & 0.026 & $1.23(0.38-3.79)$ & 0.721 \\
\hline \multicolumn{7}{|l|}{ Overall period } \\
\hline No recurrent symptoms & $30(70)$ & $95(91)$ & $5.08(1.76-15.61)$ & 0.001 & $1.96(0.45-8.40)$ & 0.361 \\
\hline No de novo heartburn & $30(70)$ & $92(88)$ & $3.29(1.24-8.86)$ & 0.014 & $1.26(0.37-4.12)$ & 0.704 \\
\hline \multicolumn{7}{|l|}{ Post-operative function } \\
\hline \multicolumn{7}{|c|}{ Minimal issues in prior 4 weeks with ${ }^{\mathrm{a}}$ : } \\
\hline Fatigue & $19(44)$ & $72(69)$ & $2.91(1.32-6.53)$ & 0.005 & $1.07(0.37-2.90)$ & 0.903 \\
\hline Daily duties & $27(63)$ & 93 (89) & $5.10(1.90-14.30)$ & $<0.001$ & $0.38(0.07-1.91)$ & 0.253 \\
\hline Leisure activities & $26(60)$ & $97(93)$ & $10.35(3.47-35.46)$ & $<0.001$ & $12.19(2.87-59.83)$ & 0.001 \\
\hline Maintaining relationships & $40(93)$ & $99(95)$ & $1.64(0.13-14.91)$ & 0.629 & - & - \\
\hline Changes in mood & $30(70)$ & $92(88)$ & $3.59(1.33-9.90)$ & 0.006 & $1.13(0.25-4.68)$ & 0.867 \\
\hline \multicolumn{7}{|c|}{ Minimal issues at present with ${ }^{\mathrm{a}}$ : } \\
\hline Mobility & $36(84)$ & $89(86)$ & $1.23(0.39-3.60)$ & 0.796 & - & - \\
\hline Self-care & $41(95)$ & $101(97)$ & $2.45(0.17-34.83)$ & 0.581 & - & - \\
\hline Usual activities & $32(74)$ & $96(92)$ & $4.65(1.50-15.46)$ & 0.004 & $0.75(0.12-4.62)$ & 0.749 \\
\hline Pain/discomfort & 34 (79) & $95(91)$ & $3.56(1.08-12.20)$ & 0.020 & $0.75(0.10-4.76)$ & 0.765 \\
\hline Anxiety/depression & 34 (79) & 93 (89) & $2.18(0.68-6.71)$ & 0.164 & - & - \\
\hline \multicolumn{7}{|l|}{ Self-perception } \\
\hline High opinion of health ${ }^{b}$ & $12(28)$ & $69(66)$ & $5.16(2.24-12.58)$ & $<0.001$ & 3.87 (1.39-11.23) & 0.010 \\
\hline \multicolumn{7}{|l|}{ Clinical outcomes } \\
\hline \multicolumn{7}{|l|}{ 30-day outcomes } \\
\hline No hospital-recorded & $33(77)$ & $96(92)$ & $4.11(1.29-13.84)$ & 0.009 & $4.31(0.93-20.81)$ & 0.063 \\
\hline \multicolumn{7}{|l|}{ 30-day complications } \\
\hline No A\&E re-attendance & $37(86)$ & $96(92)$ & $1.94(0.52-6.87)$ & 0.353 & - & - \\
\hline No readmission & $42(98)$ & $101(97)$ & $0.80(0.01-10.33)$ & 1.000 & - & - \\
\hline \multicolumn{7}{|l|}{ Length of stay } \\
\hline$\leq 1 \mathrm{~d}$ & $22(51)$ & $43(41)$ & - & 0.632 & - & - \\
\hline $2-7 d$ & $14(33)$ & $48(46)$ & & & & \\
\hline$>7 d$ & $7(16)$ & 13 (13) & & & & \\
\hline
\end{tabular}

Statistically significant values $(p<0.05)$ are given in bold

Data are $n(\%)$ unless otherwise stated

a Response of "no problems"/"slight problems"

b Numerical rating score $\geq 8 / 10$

Where the patient-reported and hospital-recorded 30-day complication rates differed for a patient, it was 5.17 times more likely [95\% CI $(2.12-15.15), p<0.001]$ that patient- reported complications were present (without a hospital record) than hospital-recorded complications (without being reported by the patient). 
Table 4 McNemar odds ratio for paired hospital-recorded and patient-reported 30-day complication rates $(\mathrm{n}=146)$

\begin{tabular}{|c|c|c|c|c|}
\hline & \multicolumn{2}{|c|}{ Hospital-recorded 30-day complications } & \multirow{2}{*}{$\frac{\text { McNemar OR }(95 \% \mathrm{CI})}{5.17(2.12-15.15)}$} & \multirow{2}{*}{$\frac{p \text { value }}{<0.001}$} \\
\hline & Absent & Present & & \\
\hline \multicolumn{5}{|c|}{ Patient-reported 30-day complications } \\
\hline Absent & 98 & 6 & & \\
\hline Present & 31 & 11 & & \\
\hline
\end{tabular}

\section{Discussion}

The purpose of this study was to determine the relationship of clinical outcome measures and PROMs with post-surgery satisfaction in a cohort of cholecystectomy patients within a 3-month post-operative period. PROMs were identified as important considerations alongside more traditional clinical outcome measures, which were not independently associated with satisfaction in this cohort. Of the PROMs independently associated with higher post-cholecystectomy satisfaction, a return to normal leisure activities showed the strongest relationship, followed by the absence of wound pain, and high ratings of current health. This suggests that traditional clinical outcome measures fail to capture aspects important to satisfaction in cholecystectomy patients, and that the determination of PROMs can provide depth in understanding the post-operative recovery of these patients.

We reported a high overall satisfaction rate $(96 \%)$ following cholecystectomy in respondents (with $71 \%$ reporting being "very satisfied"), which is comparable to rates $\geq 88 \%$ reported in other studies of cholecystectomy patients [10, 14-16]. In a high-volume centre such as ours [17], this surgical experience can have substantial benefits in surgical outcomes, particularly for high-risk patients. However, the association between PROMs and hospital volume requires further clarification, and so the clinical outcomes and PROMs reported here may not be generalisable to lower-volume hospitals. This high satisfaction may also be the result of volunteer bias from those with positive experiences [18], although the repeated attempts to contact patients aimed to minimise the impact this may have had [19]. Furthermore, respondents were found to be of a significantly higher age than non-respondents. This is concerning as older age has been found to correlate with higher post-surgical satisfaction [20, 21], hypothesised to be due to lower expectations and societal pressures [22]. This could have led to an overstated satisfaction rate, although studies on cholecystectomy patients have not found such an effect [23, 24].

We also identified a large difference in patient-reported and hospital-recorded 30-day complication rates (28.8 vs. $11.6 \%$ ) which was significant [McNemar OR: 5.17, 95\% CI (2.12-15.15), $p<0.001]$. This is the first time that these complication rates have been compared using paired analysis in cholecystectomy patients, and our results agree with a previous study [25] which reported a similarly high discrepancy between surgeon and patient-reported complications in all type surgical patients. This warrants further investigation of the nature and outcome of these patientreported complications and raises questions on whether complication rates evaluated only through hospital records adequately reflect the true complication rate experienced by patients. It would also be important to evaluate whether steps to ensure these patient-reported complications are appropriately addressed in the future. This could provide another potential avenue to improve post-surgery satisfaction in cholecystectomy patients.

The greatest strength of this study was the comprehensive and complete clinical information previously obtained for each patient. This allowed evaluation of the relationship between these surgical outcomes and subsequent PROMs and also highlights the flaws in the sole use of hospital records to investigate these outcomes. The patient-reported complications, such as wound infections, were far higher in comparison to hospital records. Therefore, surgeons may be inadvertently underestimating these risks when informing patients. In addition, the questionnaire used was a composite of three questionnaires previously utilised (two of which have been validated [8,9]) and so this increases the comparability of the PROMs reported. In addition, the response rate $(63 \%)$ to the questionnaire was higher than average for medical postal surveys [26], and this is similar to other such postal questionnaires performed within several months of cholecystectomy [27]. As response rates for postal questionnaires are often poor [28], we attempted to contact each participant before or after they received their questionnaire (with alternative telephone interviews) to minimise non-response and subsequent bias. However, this will likely still have impacted the results, given respondents were significantly older and had worse pre-operative health in terms of ASA classification.

There are several limitations in this study that should be considered. In addition to the potential biases already discussed, the questionnaire was conducted at a single point in time. As this was retrospective, it remains difficult to infer causality regarding associations identified. Furthermore, there was no standardisation regarding time to 
questionnaire completion between patients due to practical limitations of patient availability, and so respondents ranged from 1- to 3-month post-cholecystectomy. While this allowed comparison satisfaction between patients of different post-surgical periods, there was no prior or followup questionnaire, and so we cannot determine any progression in PROMs within the sample. As there can be substantial improvements in HRQoL within that time period [29], this could have had a confounding impact on the PROMs. However, this was not observed within our sample (Table 2), which could be expected within a routinely day-case procedure. Furthermore, few (15\%) of patients reported recurrent symptoms compared to other studies reporting recurrence in up to $40 \%$ of patients [10, 30, 31]. However, as these studies involved longer periods of follow-up, patients may yet develop symptoms in the future. Finally, while the cohort was of a good size and the majority of patients reported positive experiences, this limited investigation of more negative outcomes. This was particularly evident in the small number of non-satisfied patients $(n=6)$, and so with a higher response rate, or an increased sample size, we could have improved statistical power.

Previous research into post-cholecystectomy satisfaction has predominantly focussed upon the impact of post-operative symptoms (such as pain [14, 15] or recurrent symptoms [10]), as opposed to the impact of cholecystectomy on patients' daily lives. This is striking given the return to leisure activity showed the strongest independent association with satisfaction. Just one study has investigated the independent association between satisfaction and PROMs in this context [15], and those findings also support the absence of wound pain as being independently associated with positive opinions post-cholecystectomy.

The utility of PROMs to evaluate more complex surgical procedures remains undetermined; however, routine collection of post-operative PROMs data has already been implemented within the NHS in the context of hernia repair, hip and knee replacement, and varicose veins [4]. Therefore, there is a clear justification that assessment of post-cholecystectomy outcomes should also be supplemented by the use of PROMs in clinical practice order to facilitate further evaluation and improvement in patient care. With appropriate consideration of patientspecific factors, these could have a supportive role in informing treatment choice, and in monitoring patient recovery. In addition, while we cannot have confidence in inferences about causality in these associations, these factors nonetheless represent possible avenues to address in further improving the satisfaction rate in cholecystectomy patients. Although returning to leisure activity and opinions of current health are likely multifactorial in nature, patientreported wound pain in particular represents a specific and feasible target for intervention. Further research could investigate whether steps to improve these factors could include: identifying and addressing barriers to the return to leisure activity in early post-cholecystectomy recovery, and ensuring adequate post-operative analgesia. The latter would need balanced against the side-effect profile of the analgesic regimen.

In conclusion, PROMs are the major determinants of patient satisfaction following cholecystectomy, with the return to leisure activity showing the strongest association. Therefore, when assessing outcomes following cholecystectomy, the measurement of clinical outcomes alone is inadequate and should be supplemented by the use of PROMs. Furthermore, this research also highlights a potential need for further steps to encourage return to normal activities and to ensure adequate post-operative analgesia to improve patient satisfaction.

Acknowledgements None.

\section{Compliance with ethical standards}

\section{Conflicts of interest None.}

Open Access This article is distributed under the terms of the Creative Commons Attribution 4.0 International License (http://crea tivecommons.org/licenses/by/4.0/), which permits unrestricted use, distribution, and reproduction in any medium, provided you give appropriate credit to the original author(s) and the source, provide a link to the Creative Commons license, and indicate if changes were made. 


\section{Appendix}

\section{Appendix 1-Questionnaire}

Name:

Date of birth:

\section{After the operation:}

Did you have any 30 day complications?
Within 1 month after the operation, did you have complications
such as a wound infection?
Within 1 month after the operation, did you have a fever?
Within 1 month after the operation, did you have any
prolonged wound pain?
Did you have any other complications?
Later after the operation, did you have the same abdominal
symptoms that you experienced before the operation?
Did you have any pain in the scar after the operation?
Did you have heartburn as a new symptom?

How satisfied are you with the outcome of the operation?
C very satisfied
C Satisfied
C No effect
E Worse
$\mathbb{C}$ Do not know

During the past four weeks, to what extent have you had problems with the following?
a). Increased tiredness, lack of energy?
$\mathbf{C}_{\text {Not at all }} \quad \mathrm{C}_{\text {A little bit }} \quad \mathrm{C}_{\text {Moderately }} \quad \mathrm{C}_{\text {Quite a bit }} \quad \mathrm{C}_{\text {Extremely }}$

b). Inability to carry out daily duties such as housework, care for dependents, job or school related activities?
C Not at all
E A little bit
C Moderately
C Quite a bit
C Extremely

c). Inability to take part in leisure activities such as sports, hobbies, or other things you enjoy doing?
C Not at all
C A little bit
C Moderately
C Quite a bit
C Extremely

d). Inability to maintain relationships with other persons such as family, friends or the community?
C. Not at all
C A little bit
C Moderately
C Quite a bit
C Extremely
e). Changes in mood such as feeling sad, depressed, angry or worried?
C Not at all
C A little bit
C Moderately
C Quite a bit
C Extremely 
Overall, how much was your gallstone condition a problem for you?
[ Not at all
E A little bit
C Moderately
C Quite a bit
Extremely

Any other concerns, comments, or suggestions you wish to add?

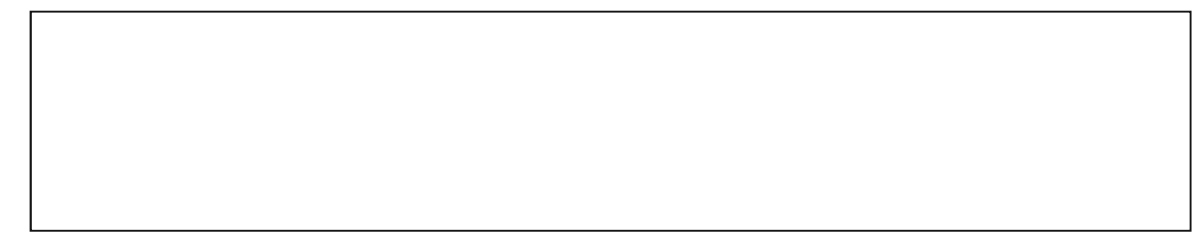

Can you rate your ability to carry out your daily tasks for the following five statements please?

Mobility

C I have no problems in walking about

[ I have slight problems in walking about

C I have moderate problems in walking about

C I have severe problems in walking about

C I am unable to walk about

\section{Self-Care}

C I have no problems washing or dressing myself

C I have slight problems washing or dressing myself

C I have moderate problems washing or dressing myself

C I have severe problems washing or dressing myself

E I am unable to wash or dress myself

\section{Usual activities}

C I have no problems doing my usual activities.

I have slight problems doing my usual activities.

C I have moderate problems doing my usual activities.

[ I have severe problems doing my usual activities.

C I am unable to do my usual activities.

\section{Pain/discomfort}

C I have no pain or discomfort.

C I have slight pain or discomfort.

C I have moderate pain or discomfort.

C I have severe pain or discomfort.

[ I have extreme pain or discomfort. 
Anxiety/Depression
C I am not anxious or depressed.
C I am slightly anxious or depressed.
[ I am moderately anxious or depressed.
C I am severely anxious or depressed.
C I am extremely anxious or depressed.

Numeric Rating Scale (NRS)

We would like you to indicate how good or bad your own health is today, in your opinion, on a scale from 0 (the worst imaginable health state) to 10 (the best imaginable health state).
$\mathbb{C}_{0} \mathbf{E}_{1}$
C 2
$\mathrm{C}_{3} \mathrm{C}_{4}$
C 5
$\mathrm{C}_{6} \mathrm{C}_{7} \mathrm{C}_{8}$
C 9
C 10

You have now completed the questionnaire; we would like to thank you for your help and participation.

\title{
Appendix 2-Informed Consent Form
}

\author{
Dear Sir / Madam,
}

\section{"Patient Reported Outcomes and Patient Experience Survey Following Cholecystectomy"}

You are invited to take part in our study to evaluate;

1) Your opinions are relating to your care as an inpatient at the Royal Infirmary of Edinburgh.

2) How you rate the outcome of the operation.

\section{What does this involve?}

If you do decide to take part, we would ask you to complete the included questionnaire (to the best of your ability) and return it via post. We would estimate the questionnaire would take you $5-10$ minutes to complete. We will follow up any unreturned questionnaires with a telephone call and there is an option to complete this as a telephone questionnaire (see telephone number below).

\section{Are there any risks to taking part?}

We do not foresee any risk to you taking part. However, we would ask that you would return this questionnaire to us via post, which would require you to supply a stamp.

\section{Are there any benefits to taking part?}

We would not expect that you would personally experience any direct benefits. However, we hope that your answers will help us to improve the care for people having similar surgery in the future.

\section{How will we keep the information safe?}

We will take the following steps to ensure that your information is confidential. Any information that you provide will be anonymized and stored on a password protected network, the access to which will be limited to only those involved in the project. The information collected will be aggregated and analyzed. This may be shared with others or published. All data will be anonymized to ensure that the answers you give cannot be traced back to you.

\section{What are your rights if you take part?}

The decision to take part is entirely up to you. However, you may wish to discuss this with your family, friends, or others. Taking part in this study is voluntary. You have the right to not take part, or to leave the study at any point if you wish. This would have no consequences, and would not affect your future care or your relationship with the staff.

\section{Any questions or problems?}

If you have any questions not answered here about the study or the questionnaire, or have any problems, please do not hesitate to contact:

Miss Kathryn Boyce MBChB, MRCS. Clinical Research Fellow, Department of Clinical Surgery, University of Edinburgh. Royal Infirmary of Edinburgh. Extension 23631. 


\section{References}

1. Peery A, Dellon E, Lund J et al (2012) Burden of gastrointestinal disease in the United States: 2012 update. Gastroenterology 143:1179-1187

2. Portincasa P, Moschetta A, Petruzzelli M et al (2006) Gallstone disease: Symptoms and diagnosis of gallbladder stones. Best Pract Res Clin Gastroenterol 20:1017-1029

3. National Institute for Clinical Excellence Interventional procedure guidance 508: Single-incision laparoscopic cholecystectomy, London, National Institute for Clinical Excellence, 2010

4. Devlin N, Appleby J (2010) Getting the most out of PROMS. The King's Fund, London

5. Berwick D (1997) Medical associations: guilds or leaders? BMJ 314:1564-1565

6. Chow A, Mayer EK, Darzi AW, Athanasiou T (2009) Patientreported outcome measures: the importance of patient satisfaction in surgery. Surgery 146:435-443

7. National Institute for Clinical Excellence Patient experience in adult NHS services: improving the experience of care for people using adult NHS services.Clinical Guidance 138, National Institute for Clinical Excellence, 2012

8. Janssen M, Pickard A, Golicki D et al (2013) Measurement properties of the EQ-5D-5L compared to the EQ-5D-3L across eight patient groups: a multi-country study. Qual Life Res 22:1717-1727

9. Chen T, Landmann M, Potter J et al (2006) Questionnaire to aid priority and outcomes assessment in gallstone disease. ANZ J Surg 76:569-574

10. Lill S, Rantala A, Karvonen J et al (2014) Elective laparoscopic cholecystectomy for symptomatic uncomplicated gallstone disease: do the symptoms disappear? Surg Endosc 28:1816-1820

11. Gupta D, Lis C, Rodeghier M (2013) Can patient experience with service quality predict survival in colorectal cancer? J Healthc Qual 35:37-43

12. Gurland B, Merlino J, Sobol T et al (2013) Surgical complications impact patient perception of hospital care. J Am Coll Surg 217:843-849

13. Kennedy G, Tevis S, Kent K (2014) Is there a relationship between patient satisfaction and favorable outcomes? Ann Surg 260:592-600

14. Vetrhus M, Berhane T, Soreide O et al (2005) Pain persists in many patients five years after removal of the gallbladder: observations from two randomized controlled trials of symptomatic, noncomplicated gallstone disease and acute cholecystitis. J Gastrointest Surg 9:826-831

15. Lamberts M, Den Oudsten B, Gerritsen J et al (2015) Prospective multicentre cohort study of patient-reported outcomes after cholecystectomy for uncomplicated symptomatic cholecystolithiasis. Br J Surg 102:1402-1409

16. Van Boxel G, Hart M, Kiszely A et al (2013) Elective day-case laparoscopic cholecystectomy: a formal assessment of the need for outpatient follow-up. Ann R Coll Surg Engl 95:561-564

17. Harrison E, O'Neill S, Meurs T et al (2012) Hospital volume and patient outcomes after cholecystectomy in Scotland: retrospective, national population based study. BMJ 344:e3330

18. Mazor K, Clauser B, Field T et al (2002) A demonstration of the impact of response bias on the results of patient satisfaction surveys. Health Serv Res 37:1403-1417

19. Barron D, West E, Reeves R et al (2014) It takes patience and persistence to get negative feedback about patients' experiences: a secondary analysis of national inpatient survey data. BMC Health Serv Res 14:153

20. Cohen G (1996) Age and health status in a patient satisfaction survey. Soc Sci Med 42:1085-1093

21. Sørlie T, Sexton H, Busund R et al (2000) Predictors of satisfaction with surgical treatment. Int J Qual Health Care 12:31-40

22. Bowling A (2002) An "inverse satisfaction law"? Why don't older patients criticise health services? J Epidemiol Community Health $56: 482$

23. Mort E, Guadagnoli E, Schroeder S et al (1994) The influence of age on clinical and patient-reported outcomes after cholecystectomy. J Gen Intern Med 9:61-65

24. Lill S, Rantala A, Vahlberg T et al (2011) Elective laparoscopic cholecystectomy: the effect of age on conversions, complications and long-term results. Dig Surg 28:205-209

25. Visser A, Ubbink D, Gouma D et al (2014) Surgeons are overlooking post-discharge complications: a prospective cohort study. World J Surg 38:1019-1025. doi:10.1007/s00268-013-2376-z

26. Asch D, Jedrziewski M, Christakis N (1997) Response rates to mail surveys published in medical journals. J Clin Epidemiol 50:1129-1136

27. Jones K, Burney R, Peterson M et al (1998) Measuring healthstatus improvement after surgery: experience with the SF-36. Semin Nurse Manag 6:139-143

28. Edwards P, Roberts I, Clarke $M$ et al (2002) Increasing response rates to postal questionnaires: systematic review. BMJ 324: $1183-1185$

29. Scarpa M (2011) Health-related quality of life outcomes after cholecystectomy. World J Gastroenterol 17:4945-4951

30. Jaunoo S, Mohandas S, Almond L (2010) Postcholecystectomy syndrome (PCS). Int J Surg 8:15-17

31. Lamberts M, Lugtenberg M, Rovers M et al (2013) Persistent and de novo symptoms after cholecystectomy: a systematic review of cholecystectomy effectiveness. Surg Endosc 27:709-718 